\title{
Serum concentration of sex hormone-binding globulin in healthy volunteers and patients with breast cancer stratified by sex and age
}

\author{
SE JUNG PARK ${ }^{1}$, TAE SOO KIM ${ }^{1}$, KYU HYUN PARK ${ }^{1}$, WOO SUN KWON ${ }^{1}$ and JIN JU KIM ${ }^{2}$ \\ ${ }^{1}$ Song Dang Institute for Cancer Research, Yonsei University College of Medicine, Seoul 03722; \\ ${ }^{2}$ Department of Laboratory Medicine, Inha University College of Medicine, Incheon 22332, Republic of Korea
}

Received November 8, 2019; Accepted March 5, 2020

DOI: $10.3892 / \mathrm{ol} .2020 .11549$

\begin{abstract}
The objective of the present study was to compare sex hormone-binding globulin (SHBG) levels according to sex (healthy male and female volunteers) and age to determine reference values. Serum SHBG expression levels in patients with breast cancer with different tumor burden states were also determined. A total of 109 samples were obtained from 34 patients in 3 different disease states (non-tumor, localized tumor and systemic metastasis) during follow-up. A sandwich ELISA was conducted to measure SHBG, cancer antigen (CA)15-3 and CA125 expression levels. Wilcoxon rank-sum tests were performed on non-normally distributed data and an unpaired t-test was used for normally distributed variables. SHBG expression levels were higher in females compared with males $(\mathrm{P}<0.0001)$. When SHBG expression levels were compared by sex, the difference was maintained in the age groups $<30,30-39$ and $\geq 50$ years, but not in the 40-49 years group. In males, SHBG expression levels increased until the age of 49 and then decreased $(\mathrm{P}=0.01)$. In females, SHBG expression levels exhibited a decreased trend until the age of $49(\mathrm{P}=0.66)$. In patients with breast cancer, the SHBG expression levels revealed a decreasing trend after the age of 50 , which was different compared with the healthy females. There was a decreasing trend of SHBG expression levels from pre-menopause to post-menopause healthy volunteers $(\mathrm{P}=0.74)$. CA15-3 $\left(\mathrm{r}^{2}=0.07 ; \mathrm{P}=0.59\right)$ and CA $125\left(\mathrm{r}^{2}=-0.18 ; \mathrm{P}=0.17\right)$ levels did not exhibit any significant correlation with SHBG expression levels. There was a significant difference in the SHBG expression levels between male and female healthy volunteers. SHBG expression levels also revealed different patterns between healthy female volunteers and female patients with
\end{abstract}

Correspondence to: Professor Jin $\mathrm{Ju}$ Kim, Department of Laboratory Medicine, Inha University College of Medicine, 27 Nam-Ku Inhang-Ro, Incheon 22332, Republic of Korea E-mail: jkimmd@inha.ac.kr

Key words: sex hormone-binding globulin, reference value, healthy volunteers, breast cancer breast cancer $\geq 50$ years of age. The present study demonstrated that SHBG does not have value as a biomarker, but different reference values according to age and sex may aid in predicting high-risk groups for hormone-dependent cancer and guide treatment direction for post-menopausal breast cancer.

\section{Introduction}

Sex hormone-binding globulin (SHBG) is a circulating glycoprotein composed of 373 amino acids and 3 carbohydrate chains that can bind to dihydrotestosterone, testosterone and estradiol, especially $\mathrm{C} 18$ or $\mathrm{C} 19$ and 17- $\beta$-hydroxyl groups. SHBG regulates plasma clearance and the uptake of sex hormones (1). High SHBG expression levels theoretically decrease the uptake of sex hormones. Notably, only a small percentage $(<2 \%)$ of steroids are unbound in plasma, and the remainder are primarily bound to SHBG and albumin (2). Therefore, SHBG may influence the carcinogenesis and progression of hormone-dependent types of cancer, such as prostate, ovary and breast cancer (3-5). There are conflicting reports regarding the association between serum levels of SHBG and the risk of prostate cancer development $(3,4)$. Grasso et al $(5)$ identified that the plasma SHBG expression levels in patients with prostate cancer were higher compared with those with benign hyperplasia or healthy volunteers. Moreover, circulating SHBG expression levels are higher in patients with lymph node invasion (6) and poor differentiation (7). In a previous prospective study of lung cancer development, there were no significant difference in the mean concentration of sex hormones or SHBG between patients who had lung cancer and those who did not have lung cancer (8). However, another previous study identified that SHBG concentration was also higher in patients with lung cancer (9).

Intracellular SHBG has been reported in liver, placenta, endometrial, breast and prostate cancers (7-10). Steroid-free SHBG can bind to the cell membrane and once bound, SHBG can bind to steroids with equal affinity as it does in the serum. This interaction is closely associated with estrogen sensitivity to each cell (11). Binding of estradiol to SHBG ultimately results in breast cancer cell apoptosis and growth suppression. Therefore, SHBG serves a protective role in the exposure of breast cells to estrogen (12). 
The SHBG expression levels in healthy postmenopausal females has been reported to be lower compared with premenopausal females, although the difference was not statistically significant (13). In patients aged 50-64 years, a decline of $10 \%$ was observed in SHBG expression levels compared with premenopausal females (14). In patients aged $>65$ years, $\mathrm{SHBG}$ expression levels returned to the pre-menopause level (14). SHBG expression levels were lower in patients with breast cancer compared with in controls (15). In pre-menopausal patients with breast cancer, the SHBG binding capacity is in the normal range; however, it is decreased in post-menopausal patients with breast cancer (16-18). The free fraction of estradiol is increased while SHBG exhibits relative or absolute decrement in post-menopausal patients with breast cancer (19). It has been suggested that different critical expression levels of SHBG must be determined for pre-menopause and post-menopausal females because the mean SHBG expression levels in these two groups differ (20). Murayama et al (20) identified that plasma expression levels of SHBG in postmenopausal patients with ER-positive breast cancer are higher compared with patients with ER-negative breast cancer. On the contrary, there was a considerable overlap of plasma SHBG expression levels between patients with estrogen receptor $(\mathrm{ER})^{+}$and $\mathrm{ER}^{-}$ endometrial and cervical cancer (13). The major beneficial effect of tamoxifen is that it can block estrogen at the receptor level and decrease the level of biologically active estradiol by upregulating SHBG expression (21). However, there was no significant association between SHBG expression level and treatment response in patients with breast cancer $(22,23)$. Lymph node metastasis and histological status in patients with high SHBG expression levels are similar to patients with low SHBG expression levels (24). The recurrence rate between high- and low-SHBG expression level groups was not significantly different and although the high SHBG group had longer disease-free survival times, this difference was not significant in premenopausal patients with breast cancer (20).

In the present study, SHBG reference range was determined based on sex and age (by decade) of healthy male and female volunteers. The serum SHBG expression levels of breast cancer exhibited a different trend compared to healthy female volunteers by age decade comparison.

\section{Patients and methods}

Collection of blood specimens. Peripheral blood samples were obtained from healthy volunteers (40 males and 40 females) at Inha University Hospital (Incheon, Republic of Korea) subsequent to obtaining approval, if no laboratory (routine blood test, liver function test and tumor markers) and imaging (plain X-ray and CT scan) abnormalities were observed during the regular medical check-up. Blood samples from 34 female patients with breast cancer were obtained at 109 different time points and grouped as follow: i) Group A, non-tumor state after surgery $(n=37)$; ii) Group B, localized tumor at diagnosis and during pre-operative chemotherapy $(\mathrm{n}=32)$; and iii) Group $\mathrm{C}$, systemic metastasis $(n=40)$ (Fig. 1). Patients with locally advanced breast cancers with clinical stage III with normal laboratory findings planned for pre-operative chemotherapy were enrolled. The median age was 40 years (range, 25-66 years) for the 40 healthy males, 34 years (range, 21-56 years) for the 40 healthy female volunteers and 45 years (range, 32-65 years) for the 34 female patients with breast cancer. Median follow-up duration was 14 months (range 2-48 months). The blood samples were stored at $-80^{\circ} \mathrm{C}$. Heparinized vacuum tubes and needles (BD Biosciences) were used to avoid platelet damage and venous occlusion, as in the clinical setting.

Determination of the normal range of serum SHBG. With 40 healthy male and 40 healthy female volunteer blood samples, the normal cut-off expression levels of SHBG were defined as the mean \pm 2 standard deviations (21-69 nmol/l) (25). Serum SHBG expression levels were considered positive when SHBG expression levels were out of this reference range ( $>69 \mathrm{nmol} / \mathrm{l}$, elevated; $<21 \mathrm{nmol} / 1$, decreased). Patients aged $\geq 50$ years were defined as post-menopause (26).

ELISA. A sandwich ELISA was performed to measure SHBG, cancer antigen (CA)15-3 and CA125 expression levels according to the manufacturer's instructions. Goat polyclonal antibody kit specific for SHBG (cat. no. M-0700; 1:50; Alpha Diagnostic Intl., Inc.) and monoclonal antibody kits specific for CA15-3 (cat. no. IS-F3329; 1:100; LifeSpan Biosciences, Inc.) and CA125 (cat. no. CA239T; 1:100; Calbiotech, Inc.) were used to coat 96-well microplates. Each blood sample was added to the plate and incubated for $1 \mathrm{~h}$ at room temperature. Following washing 3 times with wash buffer from the kit to remove unbound proteins, enzyme-linked antibodies in each kit specific for SHBG, CA15-3 and CA125 were added to wells and incubated for $1 \mathrm{~h}$ at room temperature and the absorbance was measured at $450 \mathrm{~nm}$. A standard curve was constructed by plotting absorbance values versus SHBG, CA15-3 and CA125 concentrations of the standards. Concentrations in the test samples were determined using this standard curve. All samples were run in triplicate. The detection limit of SHBG was $0.2 \mathrm{nmol} / 1$. Intra- and inter-assay variations of SHBG were 4.3-8.5 and 7.3-11.5\%, respectively. The dilution linearity was $102 \%$ (range $96-108 \%$ ). The upper normal range was $25 \mathrm{IU} / \mathrm{ml}$ for CA15-3 and $35 \mathrm{IU} / \mathrm{ml}$ for CA125.

Statistical analysis. The data were presented as mean \pm standard deviation. The Shapiro-Wilk test was performed to determine whether variables were normally distributed or not. An independent t-test was used to compare differences between two groups when variables were normally distributed, while Wilcoxon rank-sum tests were performed for non-normally distributed variables. One-way analysis of variance (ANOVA) with Bonferroni post-hoc analysis was used to examine differences for normally distributed variables among three groups or more. If the normality assumption was violated, Kruskal-Wallis with Dunn's post-hoc test was performed instead. The correlation between variables was estimated using Spearman's rank correlation coefficient. $\mathrm{P}<0.05$ was considered to indicate a statistically significant difference. All statistical analyses and graphing were performed using SAS software version 9.4 (SAS Institute, Inc., Cary, NC, USA) and R version 3.6.1. (27,28).

\section{Results}

Healthy volunteers and patients with breast cancer. The median age of healthy female volunteers was younger 


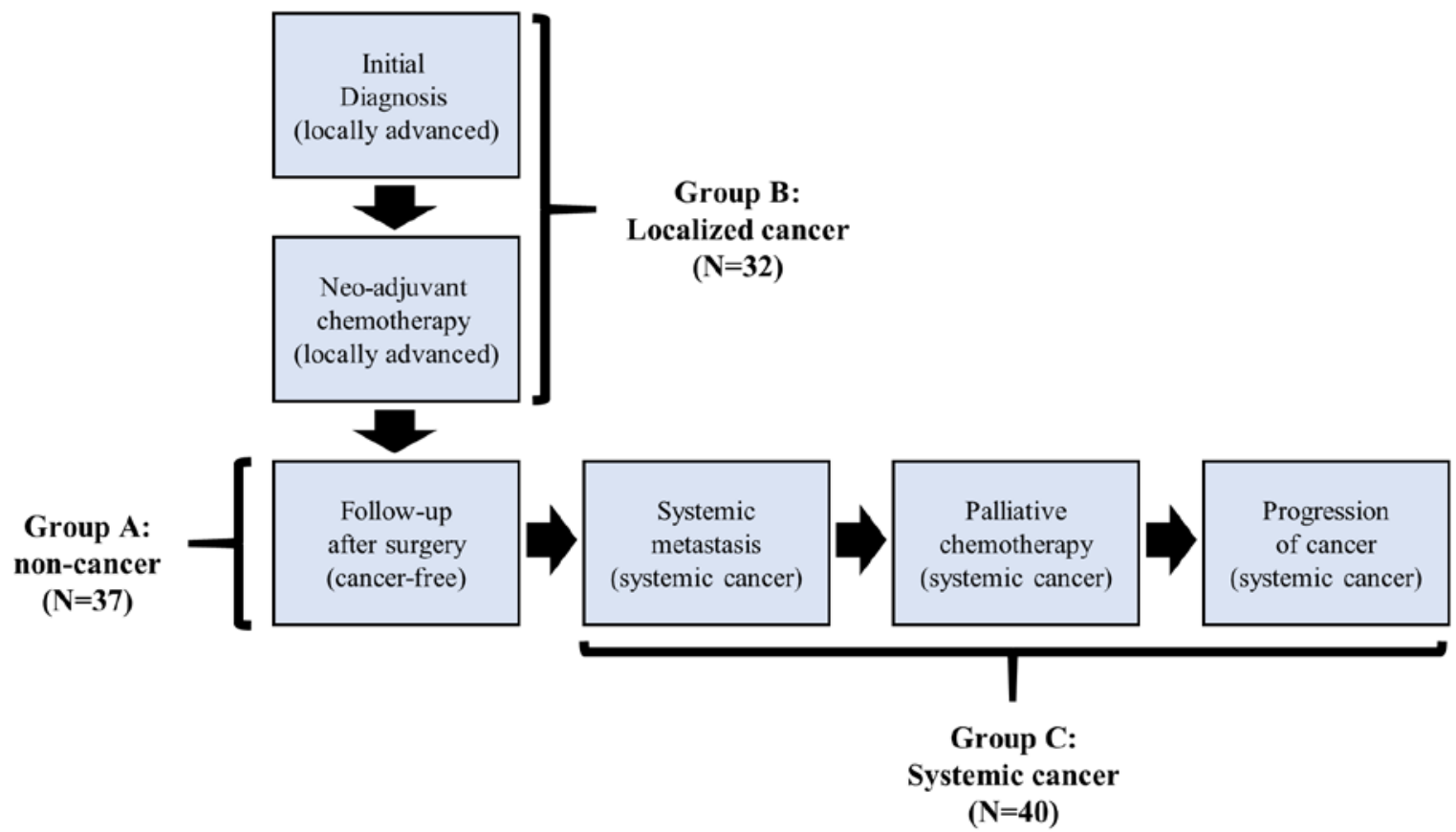

Figure 1. Different time points when blood was collected for sex hormone-binding globulin measurement during breast cancer progression with different tumor burden. (Group A) Cancer-free state with neo-adjuvant chemotherapy and surgery. (Group B) Initial diagnosis state of locally advance breast cancer. (Group C) High tumor burden state with systemic relapse after surgery.
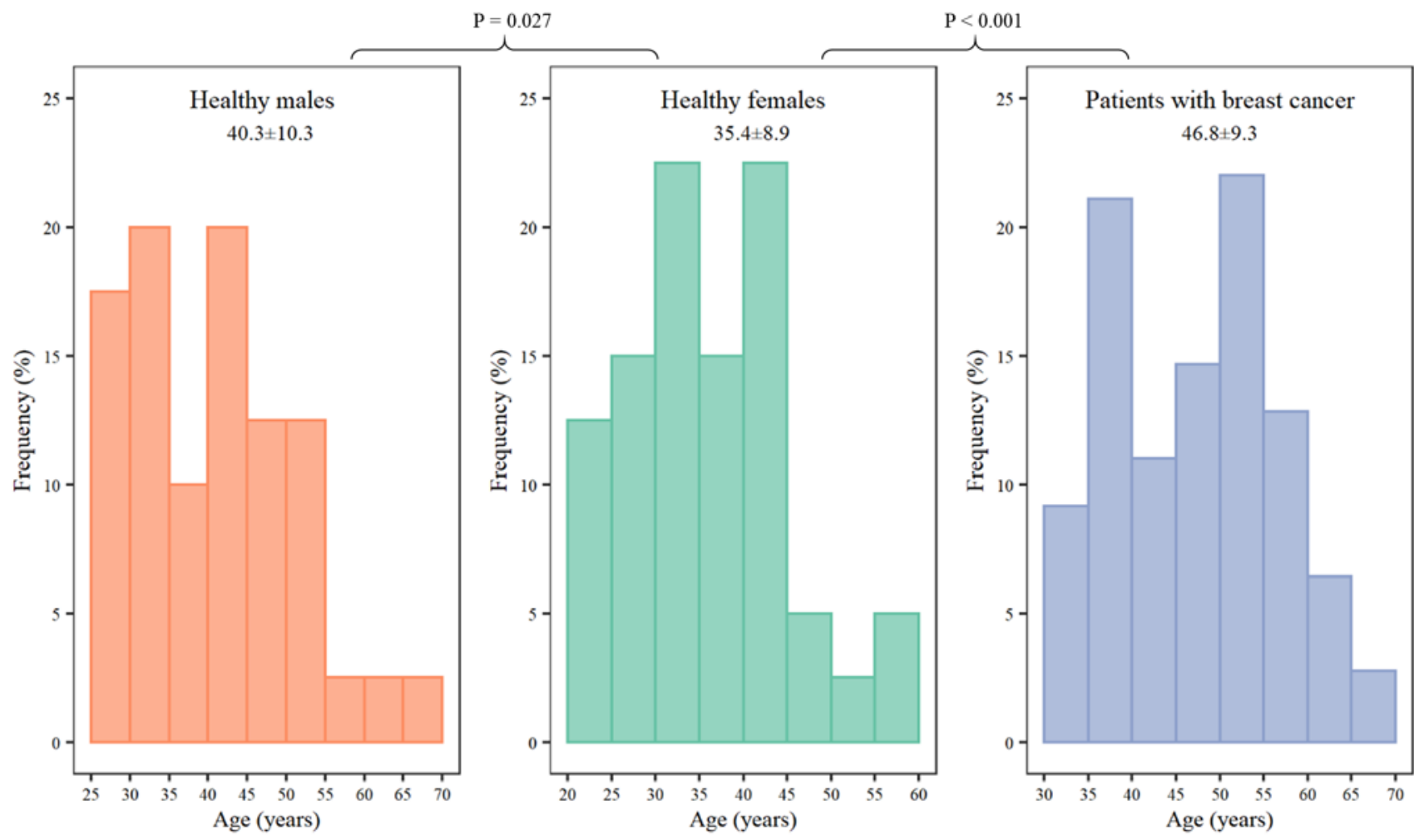

Figure 2. Age distribution of healthy males, healthy females and patients with breast cancer. Age, mean \pm standard deviation.

compared with healthy males $(\mathrm{P}=0.027)$ or patients with breast cancer $(\mathrm{P}<0.001$; Fig. 2). A total of 109 samples were obtained from patients with breast cancer in 3 different disease states (Group A, non-tumor state after surgery, $n=37$; Group B, localized tumor at diagnosis and during neo-adjuvant chemotherapy, $\mathrm{n}=32$; and Group C, systemic metastasis, $\mathrm{n}=40$ ) during a long-term follow-up (Fig. 1).

Comparison of SHBG expression levels with sex and age in healthy volunteers. The mean expression levels of SHBG 
A

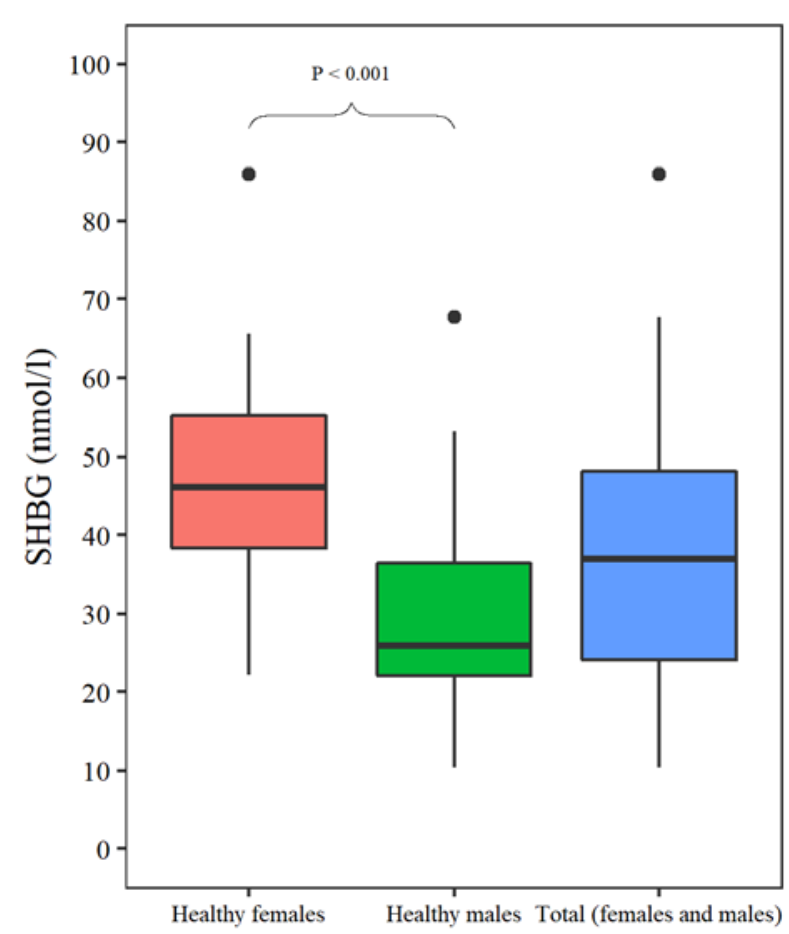

$\mathrm{C}$

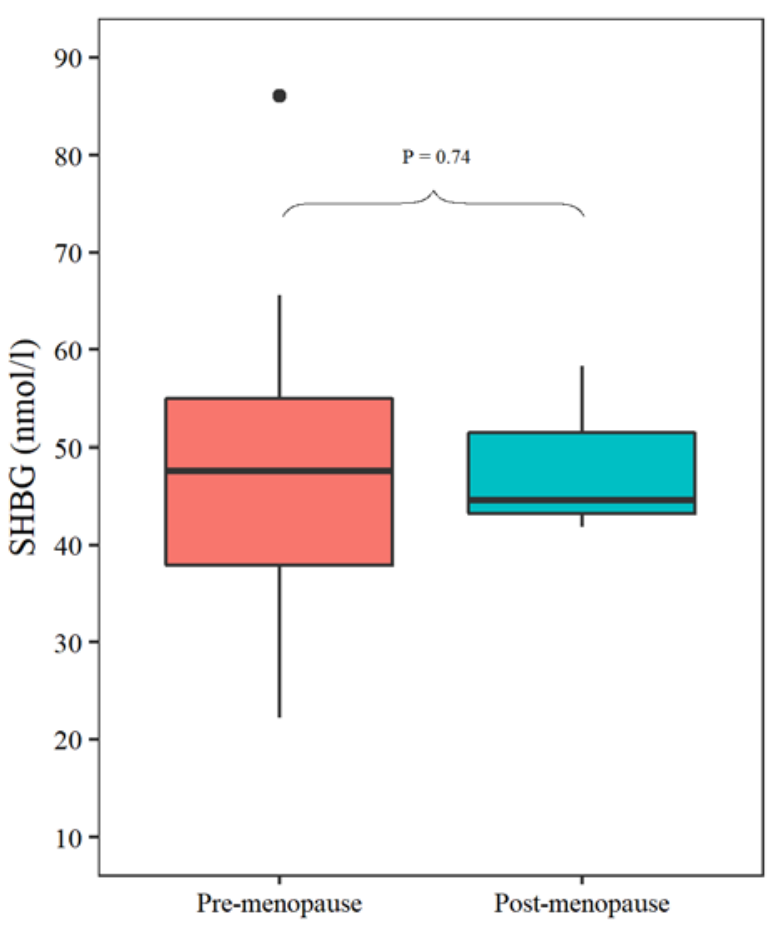

B

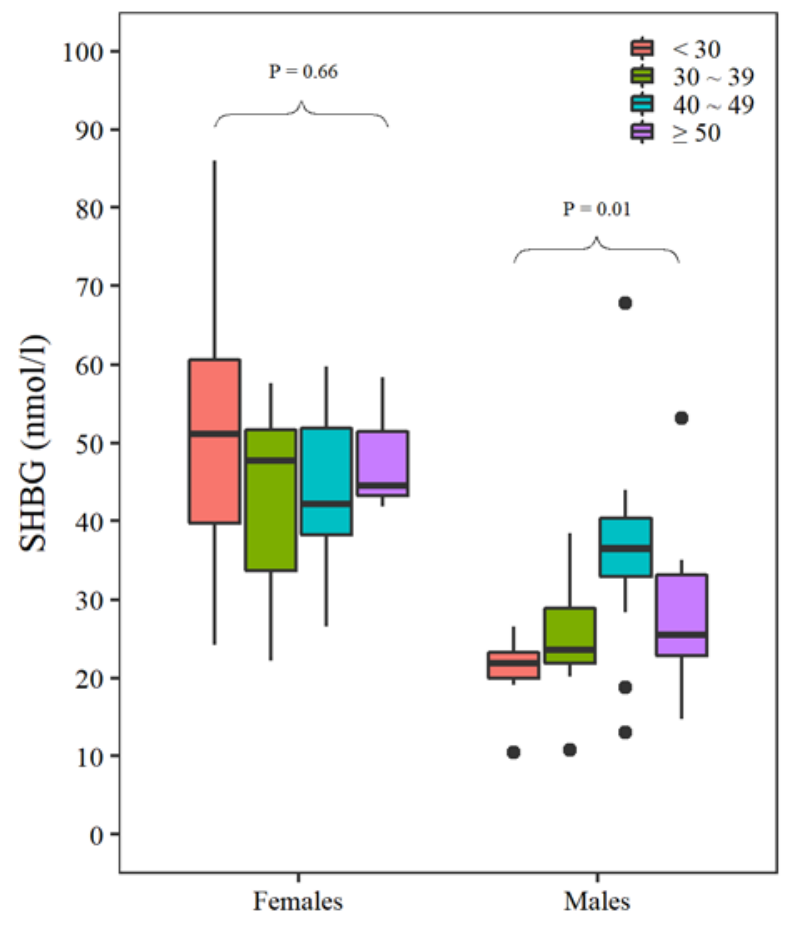

$\mathrm{D}$

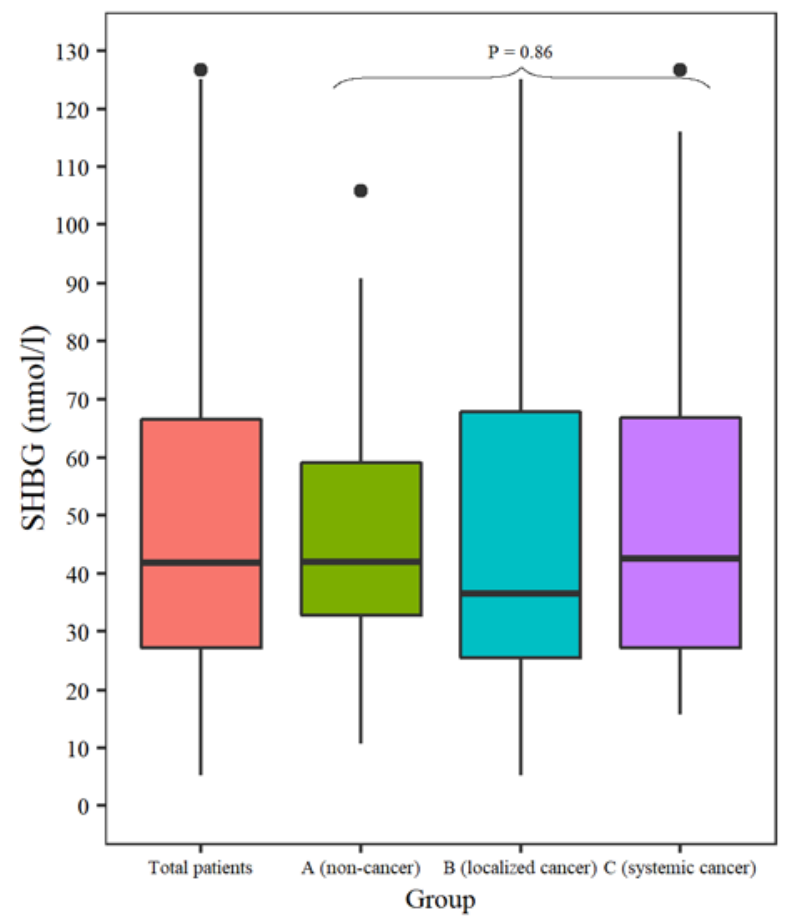

Figure 3. Comparison of SHBG expression levels in healthy volunteers and patients with breast cancer. SHBG expression levels presented by: (A) Sex (healthy females, healthy males and total healthy females and males); (B) age by decade in both healthy females and healthy males; (C) pre- and post-menopausal states of healthy females; and (D) patients with breast cancer in different cancer group with different tumor burden. SHBG, sex hormone-binding globulin.

in male and female healthy volunteers were $29.0 \pm 11.6$ and $46.4 \pm 12.8 \mathrm{nmol} / 1$, respectively. The SHBG expression levels were significantly higher in females compared with males $(\mathrm{P}<0.0001$; Fig. 3A). The difference in SHBG expression levels due to sex was compared in healthy volunteer subgroups stratified by age; the difference between sexes was maintained in people aged $<30,30-39$ and $\geq 50$ years, but not in the age group of 40-49 years (Fig. 3B) (Table I). In men, SHBG expression levels increased until the age of 49 and then decreased $(\mathrm{P}=0.01)$. In females, SHBG expression levels decreased until the age of 49. An increasing pattern in females $\geq 50$ years was identified, which was not a statistically significant difference $(\mathrm{P}=0.66$; 
Table I. Comparison of SHBG levels by sex and age in healthy volunteers.

\begin{tabular}{lcccccr}
\hline & \multicolumn{2}{c}{ Male } & & \multicolumn{2}{c}{ Female } \\
\cline { 2 - 3 } Age (years) & $\begin{array}{c}\text { Serum SHBG level, } \\
\text { mean } \pm \text { SD }\end{array}$ & Patients, $n$ & & $\begin{array}{c}\text { Serum SHBG level } \\
\text { mean } \pm \text { SD }\end{array}$ & Patients, $\mathrm{n}$ & P-value \\
\hline Range & $29.0 \pm 11.6$ & 40 & & $46.4 \pm 12.8$ & 40 & $<0.0001^{\text {a }}$ \\
$<30$ & $20.8 \pm 5.1$ & 7 & & $51.2 \pm 17.0$ & 11 & $0.0008^{\mathrm{b}}$ \\
$30-39$ & $25.6 \pm 7.9$ & 12 & & $44.4 \pm 11.3$ & 15 & $<0.0001^{\mathrm{a}}$ \\
$40-49$ & $36.6 \pm 13.1$ & 13 & & $43.8 \pm 10.8$ & 11 & $0.17^{\mathrm{b}}$ \\
$\geq 50$ & $28.9 \pm 11.7$ & 8 & & $48.3 \pm 8.8$ & 3 & $0.05^{\mathrm{b}}$ \\
P-value & & $0.01^{\mathrm{c}}$ & & & $0.66^{\mathrm{c}}$ & \\
\hline
\end{tabular}

Data were analyzed using an ${ }^{a}$ Independent t-test, a ${ }^{b}$ Wilcoxon rank sum test and a ${ }^{c}$ Kruskal-Wallis test. SHBG, sex hormone binding-globulin; $\mathrm{SD}$, standard deviation.

Table II. Comparison of SHBG levels by menopause state in female healthy volunteers.

\begin{tabular}{lccr}
\hline & Serum SHBG level, mean \pm SD & Participants, $\mathrm{n}$ & P-value \\
\hline Pre-menopause $(<50$ years $)$ & $46.3 \pm 13.2$ & 37 & $0.74^{\mathrm{a}}$ \\
Post-menopause $(\geq 50$ years $)$ & $48.3 \pm 8.8$ & 3 & \\
\hline
\end{tabular}

SHBG, sex hormone binding-globulin; SD, standard deviation; ${ }^{a}$ Kruskal-Wallis test.
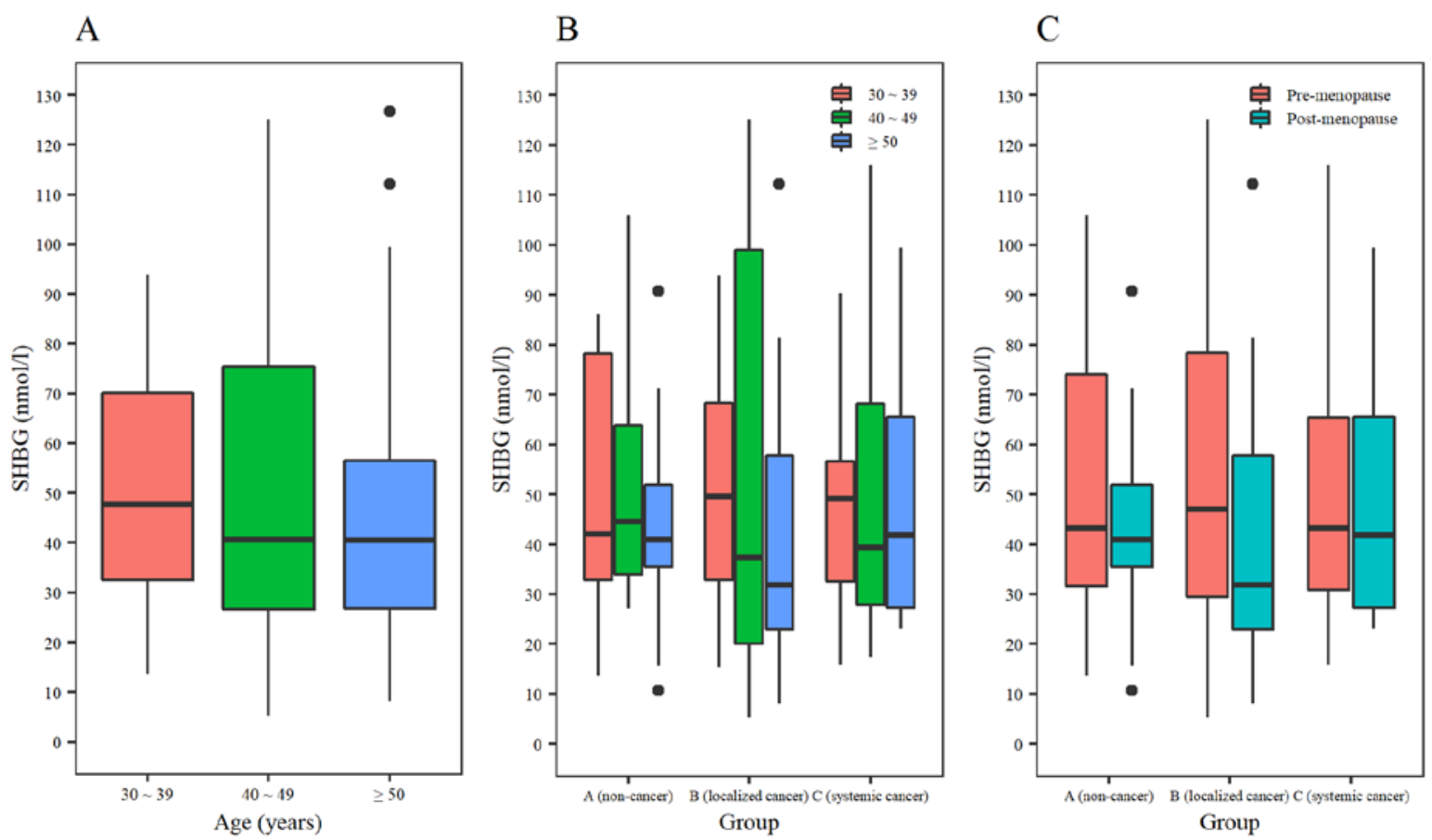

Figure 4. Comparison of SHBG expression levels in patients with breast cancer by (A) total patients by age decade, (B) by age in each cancer group with different tumor burden and (C) by menopause state in each cancer group with different tumor burden. Boxplots with maximum and minimum values. Bar represents the median value and dots are outliers. SHBG, sex hormone binding-globulin.

Fig. 3B) (Table I). In females, there was no significant difference in the SHBG expression levels between the pre-menopause group $<50$ years and the post-menopause group $\geq 50$ years (46.2 \pm 13.2 vs. $48.3 \pm 8.8$ nmol/1; P=0.74; Table II) (Fig. 3C). 
Table III. Comparison of SHBG levels by age in different cancer states.

\begin{tabular}{|c|c|c|c|c|c|c|c|}
\hline \multirow[b]{2}{*}{ Parameter } & \multicolumn{2}{|c|}{ Group A } & \multicolumn{2}{|c|}{ Group B } & \multicolumn{2}{|c|}{ Group C } & \multirow[b]{2}{*}{ P-value } \\
\hline & $\begin{array}{c}\text { Serum SHBG } \\
(\text { mean } \pm \text { SD) }\end{array}$ & Samples, n & $\begin{array}{l}\text { Serum SHBG } \\
(\text { mean } \pm \text { SD) }\end{array}$ & Samples, n & $\begin{array}{c}\text { Serum SHBG } \\
(\text { mean } \pm \text { SD) }\end{array}$ & Samples, n & \\
\hline \multicolumn{8}{|c|}{ A, age (years) } \\
\hline All patients & $47.3 \pm 23.7$ & 37 & $49.1 \pm 32.2$ & 32 & $51.4 \pm 29.0$ & 40 & $0.86^{\mathrm{a}}$ \\
\hline $30-39$ & $49.5 \pm 25.4$ & 13 & $51.9 \pm 24.2$ & 11 & $49.9 \pm 23.0$ & 9 & $0.97^{\mathrm{b}}$ \\
\hline $40-49$ & $53.3 \pm 28.0$ & 7 & $57.9 \pm 48.2$ & 7 & $51.9 \pm 32.0$ & 14 & $0.85^{\mathrm{a}}$ \\
\hline$\geq 50$ & $43.2 \pm 21.0$ & 17 & $42.4 \pm 29.1$ & 14 & $51.8 \pm 30.9$ & 17 & $0.55^{\mathrm{b}}$ \\
\hline P-value & $0.72^{\mathrm{a}}$ & & $0.59^{\mathrm{a}}$ & & $0.94^{\mathrm{a}}$ & & \\
\hline
\end{tabular}

B, menopause state

\begin{tabular}{lcccccr}
\hline Pre-menopause & $50.8 \pm 25.7$ & 20 & $54.3 \pm 34.2$ & 18 & $51.1 \pm 28.3$ & 23 \\
Post-menopause & $43.2 \pm 21.0$ & 17 & $42.4 \pm 29.1$ & 14 & $51.8 \pm 30.9$ & 17 \\
P-value & $0.33^{\mathrm{c}}$ & & $0.33^{\mathrm{d}}$ & & $0.93^{\mathrm{d}}$ & $0.68^{\mathrm{a}}$ \\
\hline
\end{tabular}

Data were analyzed using a ${ }^{\mathrm{a}}$ Kruskal-Wallis test, ${ }^{\mathrm{b}} \mathrm{ANOVA}$, an ${ }^{\mathrm{c} I n d e p e n d e n t ~ t-t e s t ~ a n d ~ a ~}{ }^{\mathrm{d}}$ Wilcoxon rank sum test. SHBG, sex hormone bindingglobulin; SD, standard deviation.

Table IV. Comparison of SHBG positivity in each decade of age in breast cancer.

\begin{tabular}{lccc}
\hline Biomarker & Sensitivity, $\%$ & Specificity, $\%$ & Accuracy, $\%$ \\
\hline Total $(\mathrm{n}=109)$ & 36 & 65 & 46 \\
$30-39(\mathrm{n}=33)$ & 35 & 54 & 42 \\
$40-49(\mathrm{n}=28)$ & 52 & 71 & 57 \\
$\geq 50(\mathrm{n}=48)$ & 26 & 71 & 42 \\
$\begin{array}{l}\text { Pre-menopause } \\
\text { (n=61) }\end{array}$ & 44 & 60 & 50 \\
$\begin{array}{l}\text { Post-menopause } \\
\text { (n=48) }\end{array}$ & 26 & 71 & 42 \\
\hline
\end{tabular}

SHBG expression levels in patients with breast cancer. Although there was a trend of increasing SHBG expression levels with larger tumor volumes, there was no statistically significant difference $(\mathrm{P}=0.86)$ in the $\mathrm{SHBG}$ expression levels between the 3 different tumor states: Group A $(n=37)$, $47.3 \pm 23.7 \mathrm{nmol} / \mathrm{l}$; Group B ( $\mathrm{n}=32), 49.1 \pm 32.2 \mathrm{nmol} / \mathrm{l}$; and Group C ( $\mathrm{n}=40), 51.4 \pm 29.0 \mathrm{nmol} / \mathrm{l}$ (Fig. 3D). SHBG expression levels were compared by age in patients with breast cancer regardless of cancer state, and these levels exhibited a decreasing trend which revealed an increasing trend in healthy females (Fig. 4A). SHBG expression levels were compared by age between the 3 groups; there was no significant difference in Group A by age; however, Groups B and C exhibited a decrease in expression in the $\geq 50$ years groups (Fig. 4B). SHBG expression levels were compared between pre-menopause and post-menopause groups; the SHBG expression level exhibited a decreasing trend in post-menopausal patients compared
Table V. Comparison of sensitivity, specificity and accuracy of 3 biomarkers in breast cancer.

\begin{tabular}{lccc}
\hline Biomarker & Sensitivity, \% & Specificity, \% & Accuracy, \% \\
\hline CA15-3 & 61 & 82 & 68 \\
CA125 & 51 & 65 & 56 \\
CA15-3 and & 75 & 59 & 70 \\
CA125 & & & \\
CA15-3, CA125 & 79 & 32 & 64 \\
and SHBG & & & \\
\hline
\end{tabular}

with pre-menopausal patients, in all three groups (Table III; Fig. 4C).

Comparison of serum SHBG positivity in each decade of age in breast cancer. Using a cut-off point of the mean \pm 2 standard deviations for SHBG positivity, a sensitivity of $36 \%$, a specificity of $65 \%$ and an accuracy of $46 \%$ were identified. For the pre-menopause group, the sensitivity, specificity and accuracy were 44, 60 and 50\%, respectively. In the post-menopause group, the sensitivity, specificity and accuracy were 26, 71 and $42 \%$, respectively. When the sensitivity and specificity in each decade were evaluated in whole breast cancers, the sensitivity and specificity were as follows: 35 and 54\% for age $30-39$ years; 52 and $71 \%$ for age $40-49$ years; and 26 and $71 \%$ for age $\geq 50$ years, respectively (Table IV).

Comparison of serum positivity among SHBG, CA15-3 and CA125 in breast cancer. In 109 samples with different states of breast cancer, sensitivity, specificity and accuracy of 

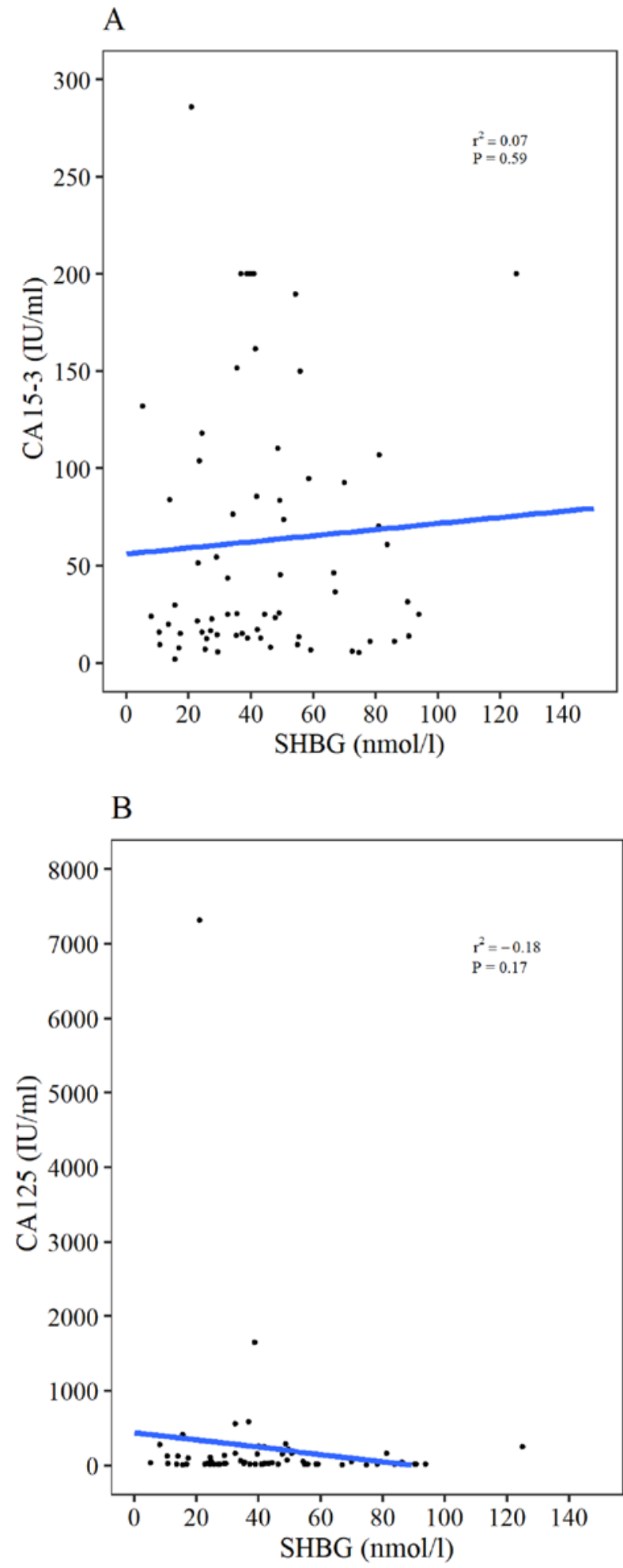

Figure 5. Correlation between SHBG and (A) CA15-3 or (B) CA125. SHBG, sex hormone binding-globulin; CA, cancer antigen.

CA15-3 and CA125 were simultaneously compared (Table V). Sensitivity increased when considering CA15-3 and CA125 together (75\%). The highest sensitivity (79\%) was obtained when all three markers were used. When SHBG was measured alongside CA15-3 or CA125 (64\%), no benefit was identified regarding accuracy with CA15-3 or CA125 (70\%) (Table V). CA15-3 and CA125 levels were moderately correlated with each other $\left(\mathrm{r}^{2}=0.54 ; \mathrm{P}<0.0001\right.$; data not shown). However, both CA15-3 $\left(\mathrm{r}^{2}=0.07 ; \mathrm{P}=0.59\right)$ and $\mathrm{CA} 125\left(\mathrm{r}^{2}=-0.18 ; \mathrm{P}=0.17\right)$ serum levels were not significantly correlated with SHBG (Fig. 5A and B, respectively).

\section{Discussion}

SHBG is a circulating glycoprotein that binds dihydrotestosterone, testosterone and estradiol; notably, its highest binding affinity is for dihydrotestosterone and testosterone, with a lower affinity for estradiol (1). As a result, an increase in SHBG serum concentration may result in lowering the percentage of unbound dihydrotestosterone, testosterone and estradiol (2). Therefore, SHBG may be a useful predictor of circulating total and bioavailable sex hormone levels (2). By contrast to healthy females, to the best of our knowledge, there has been no analysis of the SHBG expression levels in healthy males. As revealed by the present data, although there was a difference in age distribution of male to female volunteers, healthy males exhibited a lower range of SHBG expression levels compared with healthy females by age (decade of age). This indicates that different normal reference values of SHBG expression levels for males and females are needed to determine abnormal expression levels of SHBG for risk evaluation of cancer or for cancer status prediction. In males, the SHBG expression levels increased from 30 to 49 years of age and then decreased $\geq 50$ years, at which age the incidence of prostate cancer typically increases (3). This pattern was reversed in females who exhibited a decreasing trend until 49 years. SHBG expression levels increased in patients aged $>50$ years, when the incidence of breast cancer typically increases $(15,23)$.

There is controversy regarding the association between the serum levels of SHBG and the risk of prostate cancer development, which may come from fixed normal reference value regardless of sex and age. In a Japanese population, SHBG expression levels were not strongly associated with the risk of prostate cancer, except in males age $<60$ years (3). However, a previous study in Spain identified that low bioavailability of testosterone levels and high SHBG expression levels were associated with a 4.9- and 3.2-fold increase in the risk of prostate cancer, respectively (4). In localized prostate cancer, the preoperative serum SHBG expression levels were associated with prostatic extension and Gleason score (29). SHBG was not considered a biomarker for high-grade disease (30). For future prostate cancer studies, normal reference values of SHBG should reflect sex and age. In lung cancer which is non-endocrine cancer, the typical negative correlation between SHBG expression levels and total dihydrotestosterone observed in healthy volunteers and other endocrinological gynecology cancer was reversed, perhaps due to the systemic manifestation of thyrotoxicosis, chronic liver disease and disseminated cancer associated with liver metastasis $(8,9)$. In patients with cancer, different reference ranges by age, sex, cancer type (endocrinological versus non-endocrinological) and cancer stage (localized versus systemic manifestations) can be applied to clarify these controversial clinical results.

A previous study demonstrated that SHBG expression levels are higher in the first 12 days of luteal phase compared with the rest of the menstrual cycle $(31,32)$. In both pre-menopause and post-menopause groups, SHBG expression levels 
decreased with increasing weight. SHBG level was lower in single nulliparous compared with married nulliparous or parous females (31). In post-menopause, SHBG increased in the years following menopause (31). In the present study of healthy volunteers, a similar trend was revealed as SHBG expression levels exhibited a decreasing trend until age 49 and then an increasing pattern $>50$ years. Therefore, it was suggested that the SHBG expression levels must be determined for pre-menopausal and post-menopausal females separately, as the mean SHBG expression levels in these two groups were different (20). Serum SHBG expression levels are regulated by a biologically active and unbound hormone fraction, with androgens having an inhibitory effect and estrogens having a stimulatory effect on the SHBG expression levels $(1,16,33)$. High SHBG expression levels were significantly associated with decreased breast cancer risk and protective function in post-menopausal females (34). In the present study, in patients with breast cancer in Groups B and $\mathrm{C}$, the SHBG expression levels indicated a decreasing trend after age 50, although it showed an increasing trend in healthy females aged $\geq 50$ years. Although the patients in this study were mostly younger than Western patients (35), receiving mainly chemotherapy instead of hormonal treatment and the healthy volunteers were younger than the patients with cancer, the trend was similar to that in Western patients when the SHBG expression levels was compared by age $(14,15)$. SHBG expression levels were increased by estrogen but decreased by testosterone, suggesting that upregulated SHBG may be an indicator of an estrogenic environment (11). Therefore, it was suggested that SHBG expression levels were an improved predictor of hormone treatment compared with the estrogen receptor, as higher SHBG expression levels were identified in $\mathrm{ER}^{+}$patients compared with $\mathrm{ER}^{-}$patients $(20,33,36)$. Notably, the SHBG expression levels in postmenopausal patients with $\mathrm{ER}^{+}$were higher compared with patients with ER- endometrial and cervical cancer, even if the SHBG level revealed a high overlapping range between $\mathrm{ER}^{+}$and $\mathrm{ER}^{-}$groups (1). Whether the considerable overlap of SHBG expression levels between $\mathrm{ER}^{+}$and $\mathrm{ER}^{-}$gynecological cancer is due to other factors, such as heterogeneity of tumor stage, varying degree of illness or weight, needs to be studied in the future (13).

A novel biomarker should be an independent predictor of the selected outcome; it must increase the multivariable predictive accuracy of a model (24). Despite controversy surrounding the correlation of SHBG with ER status, suggesting that plasma SHBG has little value as a predictive index in breast cancer, Dimou et al (37) recently reported a potentially causal inverse association between SHBG expression levels and risk of ER positive breast cancer. In the present study, although specificity was good $\geq 50$, the sensitivity was too low in patients with breast cancer to confirm the role of SHBG as a tumor suppressor. SHBG expression levels were not correlated with known tumor markers CA15-3 or CA125. No additive effect of the biomarkers was identified using all three biomarkers for cancer prediction. A larger study using an age-specific reference value with estrogen level may resolve this issue in the future. A non-synonymous single nucleotide polymorphism in exon 8 can result in an amino acid substitution of asparaginase for aspartic acid (D356N, rs6259) in the SHBG protein (38). The asparagine allele of SHBG was associated with elevated circulating SHBG in postmenopausal females (38). This genotype may be applied in future studies as a biomarker.

Genistein not only increases SHBG expression in Hep-G2 cells, but also suppresses Hep-G2 cell proliferation (2). As genistein is an inhibitor of tyrosine-specific protein kinases, isoflavonoid may serve a role in the prevention of malignant tumors, including hormone-dependent cancers in countries with high consumption of soy products, such as Japan and Korea (2). Genotyping and diet analysis must be combined in the future to determine the protective role of SHBG in female breast and gynecological cancer.

In conclusion, there was a significant difference in the SHBG expression levels between male and female healthy volunteers. There was also a different pattern in the SHBG expression levels between female healthy volunteers and female patients with breast cancer $\geq 50$ years. Although SHBG itself cannot be used as a biomarker, different reference values stratified by age and sex may help to determine its role in predicting a high-risk group for hormone-dependent cancer, and guide treatment in post-menopausal patients with breast cancer.

\section{Acknowledgements}

Not applicable.

\section{Funding}

No funding was received.

\section{Availability of data and materials}

The datasets used and/or analyzed during the current study are available from the corresponding author on reasonable request.

\section{Authors' contributions}

JK designed the present study, interpreted the data and wrote the first draft of the manuscript. SP interpreted the data and performed the statistical analysis. TK, KP and WK performed the experiments, interpreted the data, and revising the draft. All authors read and approved the final manuscript.

\section{Ethics approval and consent to participate}

Patients provided written informed consent to participate in the present study, which was approved from The Institutional Review Board of Inha University Hospital (approval no. 10-617) and Severance Hospital, Yonsei University College of Medicine (approval no. 4-2009-0256).

\section{Patient consent for publication}

Not applicable.

\section{Competing interests}

The authors declare that they have no competing interests. 


\section{References}

1. Selby C: Sex hormone binding globulin: Origin, function and clinical significance. Ann Clin Biochem 27: 532-541, 1990.

2. Mousavi Y and Adlercreutz H: Genistein is an effective stimulator of sex hormone-binding globulin production in hepatocellular carcinoma human liver cancer cells and suppresses proliferation of these cells in culture. Steriods 58: 301-304, 1993.

3. Sawada N, Iwasaki M, Inoue M, Sasazuki S, Yamaji T, Shiazu T, Tsugane S and Japan Public Health Center-based Prospective Study Group: Plasma testosterone and sex hormone-binding globulin concentrations and the risk of prostate cancer among Japanese men: A nested case-control study. Cancer Sci 101 2652-2657, 2010

4. Garcia-Closas M, Brinton LA, Lissowsk J, Richesson D, Sherman ME, Szeszenia-Dabrowska N, Peplonska B, Welch R Yeager M, Zatonski W and Chanock SJ: Ovarian cancer risk and common variation in the sex hormone binding globulin gene: A population-based case-control study. BMC Cancer 7: 60, 2007.

5. Grasso M, Buonaguidi A, Mondina R, Borsellino G, Lania C, Banfi $G$ and Rigatti P: Plasma sex hormone binding globulin in patients with prostatic carcinoma. Cancer 66: 354-357, 1990.

6. Salonia A, Briganti A, Gallina A, Karakiewicz P, Shariat S Freschi M,Zann G, Caoitanio U, Bosi E, Rigatti P and Montorsi F: Sex hormone-binding globulin: A novel marker for nodal metastases prediction in prostate cancer patients undergoing extended pelvic lymph node dissection. Urology 73: 850-855, 2009.

7. Haapianen R, Rannikko S, Adlercreutz H and Alfthan O: Correlation of pretreatment plasma levels of estradiol and sex-hormone binding globulin-binding capacity with clinical stage and survival of patients with prostate cancer. The Prostate 8: 127-137, 1986

8. Calciano A, Khaw KT, Barrett-Connor E and Garland C: Sex hormones, sex hormone binding globulin, and lung cancer: A 12 year prospective study in a cohort of malesaged $50-79$. Br Med J 296: 1640, 1988

9. Corbishley TP, Keating JJ, Johnson PJ and Williams R: Serum concentrations of sex hormone binding globulin in lung cancer. Br Med J 293: 792-793, 1986.

10. Fortunati N, Catalano MG, Boccuzzi G and Fairia R: Sex hormone binding globulin, estradiol and breast cancer. Mol Cell Endocrinol 316: 86-92, 2010.

11. Dayalu NSL, Suresh H, Anil KB, Renuka S, Sudershan K and Amitabha R: Sex hormone binding globulin in breast cancer. Indian J Clin Biochemist 23: 25-254, 2008.

12. Catalano MG, Frairia R, Boccuzzi G and Fortunati N: Sex hormone-binding globulin antagonizes the anti-apoptotic effect of estradiol in breast cancer cells. Mol Cell Endocrinol 230: 31-37, 2005.

13. Ratajczak T, Twaddle E and Hähnel R: Sex hormone-binding globulin and estrogen receptor in endometrial and cervical cancer. Gynecol Oncol 10: 262-266, 1980.

14. Moore JW, Key TJ, Clark GM, Bulbrook RD, Allen DS, Wang DY and Pike MC: Concentrations of sex hormone-binding globulin (SHBG) in a population of normal females: Relationship to risk factors for breast cancer. Steroids 52: 391-392, 1988.

15. Adami HO, Johansson EDB, Vegelius J and Victor A: Serum concentrations of estrone, androstenedione, testosterone and sex hormone-binding globulin in postmenopausal females with breast cancer and in age-matched controls. Upsala J Med Sci 84: 259-274, 1979.

16. Moore JW, Clark GM, Bulbrook RD, Hayward JL, Murai JT, Hammond GL and Siiteri PK: Serum concentrations of total and non-protein-bound estradiol in patients with breast cancer and on normal controls. Int J Cancer 29: 17-21, 1982.

17. Toniolo PG, Levitz M, Zeleniuch-Jacquotte A, Banerjee S, Koenig KL, Shore RE, Strax P and Pasternack BS: A prospective study of endogenous estrogens and breast cancer in postmenopausal females. J Natl Cancer Inst 87: 190-197, 1995.

18. Zeleniucg-Jacquotte A, Shore RE, Koenig KL, Akhmedkhanov A, Afanasyeva Y, Kato I, Kim MY, Rinaldi S, Kaaks R and Toniolo P: Postmenopausal levels of estrogen, androgen, and SHBG and breast cancer: Longterm results of a prospective study. Br J Cancer 90: 153-159, 2004.

19. Lipworth L, Adami HO, Trichopoulos D, Carlstrom K and Mantzoros C: Serum steroid hormone levels, sex-hormone binding globulin and body mass index in the etiology of postmenopausal breast cancer. Epidemiology 7: 96-100, 1996.
20. Murayama Y, Utsunomiya J, Takahashi I, Kitamura $M$ and Tominaga T: Sex hormone binding globulin as a reliable indicator of hormone dependence in human breast cancer. Ann Surg 190: 133-138, 1979.

21. Sakai F, Cheix F, Clavel M, Colon J, Mayer M, Pommatau E and Saez S: Increases in steroid binding globulins induced by tamoxifen in patients with carcinoma of the breast. J Endocrinol 76: 219-226, 1978.

22. Wang DY, Rubend RD, Clark GMG, Moore JW and Bulbrook RD: Effects of prednisolone on sex hormone binding globulin during primary endocrine treatment of advanced breast cancer. Breast Cancer Res Treat 11: 67-70, 1988.

23. Beattie MS, Costantino JP, Cummings SR, Wickerham DL, Vogel VG, Dowsett M, Folkerd EJ, Willett WC, Wolmark N and Hankinson SE: Endogenous sex hormones, breast cancer risk, and tamoxifen response: An ancillary study in the NSABP Breast Cancer Prevention Trial (P-1). J Natl Cancer Inst 98: 110-115, 2006.

24. Kattan MW: Judging new markers by their ability to improve predictive accuracy. J Natl Cancer Inst 95: 634-635, 2003.

25. Rha SY, Yang WI, Gong SJ, Kim JJ, Yoo NC, Roh JK, Min JS, Lee KS, Kim BS and Chung HC: Correlation of tissue and blood plasminogen activation system in breast cancer. Cancer Lett 150: 137-145, 2000.

26. Park HA, Park JK, Park SA and Lee JS: Age, menopause, and cardiovascular risk factors among Korean middle-aged women: The 2005 Korea National Health and Nutrition Examination Survey. J Womens Health (Larchmt) 19: 869-876, 2010.

27. R Core Team: R: A language and environment for statistical computing. R Foundation for Statistical Computing, Vienna, 2012. http://www.R-project.org/

28. RStudio Team: RStudio: Integrated development for R. RStudio, Inc., Boston, MA, 2019. http://www.rstudio.com/

29. Lee SE, Chung JS, Han BK, Park CS, Moon KH, Byun SS, Choe GY and Hong SK: Pre-operative serum sex hormone-binding globulin as a predictive marker for extra-prostatic extension of tumor in patients with clinically localized prostate cancer. Eur J Urol 54: 1324-1332, 2008.

30. Nunzio CD, Lombardo R, Albisinni S, Gacci M and Tubaro A: Serum levels of sex hormone binding globulin (SHBG) are not predictive of prostate cancer diagnosis and aggressiveness: results from an Italian biopsy cohort. Int Braz J Urol 39: 793-799, 2013.

31. Moore JW, Key TJA, Bulvrook RD, Clark GMG, Allen DS, Wang DY and Pike MC: Sex hormone binding globulin and risk factors for breast cancer in a population of normal females who had never used exogenous sex hormones. Br J Cancer 56: 661-666, 1987.

32. Pugeat M, Cousin P, Baret C, Lejeune H and Forest MG: Sex hormone-binding globulin during puberty in normal and hyperandrogenic girls. J Pediatr Endocrinol Metab 13 (Suppl): $1277-1279,2000$

33. Plymate SR, Stutz FH and Fariss BL: Relationship between sex hormone-binding globulin and estrogen receptors in breast cancer. J Clin Oncol 2: 652-654, 1984

34. He XY, Liao YD, Zhang Y and Wang R: Sex hormone binding globulin and risk of breast cancer in postmenopausal females: A meta-analysis of prospective studies. Horm Metab Res 47: 485-490, 2015

35. Kim Z, Min SY, Yoon CS, Lee HJ, Lee JS, Youn HJ, Park HK, Noh DY, Hur MH and Korean Breast Cancer Society: The basic facts of Korean breast cancer in 2011: Results of a nationwide survey and breast cancer registry database. J Breast Cancer 17: 99-106, 2014

36. Fortunati N, Becchis M, Catalano MG, Comba A, Ferrera P, Raineri M, Berta L and Frairia R: Sex hormone-binding globulin, its membrane receptor, and breast cancer: A new approach to the modulation of estradiol action in neoplastic cells. J Steroid Biochem Mol Biol 69: 473-479, 1999.

37. Dimou NL, Papadimitriou N, Gill D, Christakoudi S, Murphy N, Gunter MJ, Travis RC, Key TJ, Fortner RT, Haycock PC, et al: Sex hormone binding globulin and risk of breast cancer: A Mendelian randomization study. Int J Epidemiol 48: 807-816, 2019.

38. Zhou JY, Shi R, Yu HL, Zheng WL and Ma WL: Association between SHBG Asp327Asn (rs6259) polymorphism and breast cancer risk: A meta-analysis of 10,454 cases and 13,111 controls. Mol Biol Rep 39: 8307-8314, 2012.

This work is licensed under a Creative Commons Attribution-NonCommercial-NoDerivatives 4.0 International (CC BY-NC-ND 4.0) License. 